macrophages, and whether they are required for this remodelling remains unclear. We have recently developed a mouse model (MacLow) where approximately $50 \%$ of macrophages are depleted and now aim to investigate whether MacLow mice would demonstrate a reduced pulmonary hypertension phenotype in response to hypoxia, when compared to non-macrophage ablated littermates.

Methods Macrophage ablation was induced in CD68-rtTA-eGFP/ tetDTA double transgenic mice (MacLow) where macrophagespecific (CD68) induction of the cytotoxic diphtheria toxin A chain (DTA) is achieved by administration of doxycycline containing chow diet (doxy-chow). Mice were divided by sex and then fed either regular or doxy-chow for 2 weeks prior to either 2 weeks exposure to hypoxia (10\% oxygen), or room air. All mice were phenotyped for $\mathrm{PH}$ by echocardiography followed by closed chest cardiac catheterisation. Heart and lung tissue were harvested for morphological, immunohistochemical and biochemical analyses.

Results Doxy-chow fed mice displayed the expected $50 \%$ reduction in macrophages (liver) compared to controls. MacLow mice with the induced ablation of macrophages were not protected from hypoxia induced pulmonary hypertension although females displayed a trend for higher RVSP after hypoxia ( $34 \mathrm{~mm} \mathrm{Hg}$ vs $29 \mathrm{~mm} \mathrm{Hg}$ ). Interestingly male MacLow mice with induced macrophage ablation displayed a spontaneous $\mathrm{PAH}$ phenotype ( $33 \mathrm{~mm} \mathrm{Hg}$ ), in normoxia, that was not further increased by hypoxia. The changes in RVSP were accompanied by appropriate changes in RVH.

Conclusion These data suggest that macrophages play a modulating role in pulmonary vascular remodelling but further work is required to explore the mechanisms involved in this phenotype, and to fully assess the change in macrophage number within the lungs of these mice.

\section{P6 MANAGING ACUTE PULMONARY EMBOLISM IN THE OUTPATIENT SETTING: INITIAL EXPERIENCES AND OUTCOMES IN A UK DISTRICT GENERAL HOSPITAL}

doi:10.1136/thoraxjnl-2011-201054c.6

J P Corcoran, J E S Park, C Goode, C W H Davies. Royal Berkshire NHS Trust, Reading, $U K$

Introduction and Objectives Outpatient (OP) management of low risk cases of pulmonary embolism (PE) can be as effective as inpatient (IP) management and reduce length of stay (LOS) (1). Concerns exist regarding the safety of this strategy, with a wide range of adverse outcomes reported (2). In our hospital a treatment

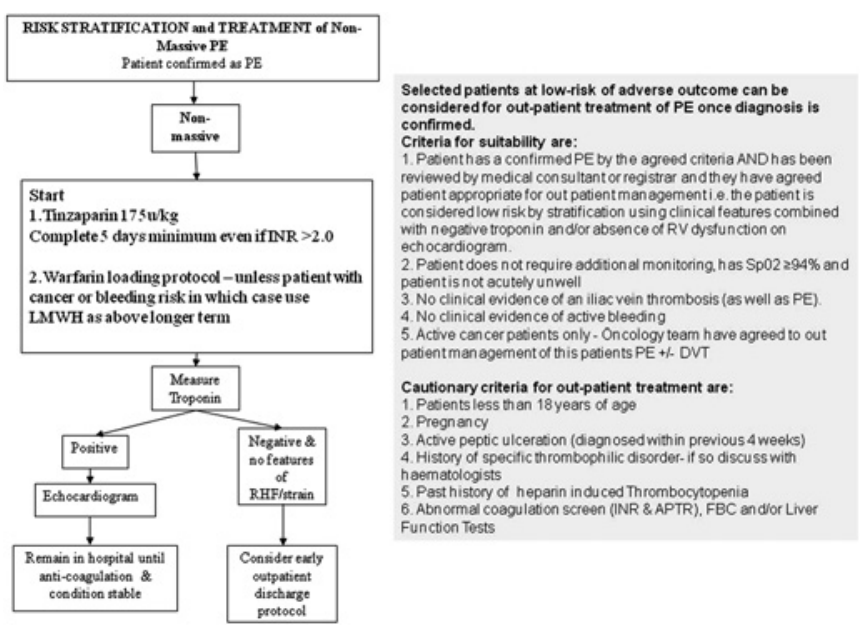

Abstract P6 Figure 1 Pathway to identify low risk individuals diagnosed with PE suitable for OP management. pathway was implemented to identify low risk individuals diagnosed with PE suitable for OP management, based on the European Society of Cardiology PE Guidelines (2008) (Abstract P6 figure 1). The aim of this study was to retrospectively review all cases of acute $\mathrm{PE}$ in a 6-month period, to determine appropriateness of management as OP and assess LOS, venous thromboembolism (VTE) recurrence and anticoagulation related adverse events.

Methods Episodes of PE occurring between January and June 2010 were identified through clinical coding (ICD-10: I26). Clinical notes were reviewed and data collected for LOS, time to diagnostic investigation, risk stratification (troponin, echocardiography and adverse clinical features), IP/OP management, recurrence of VTE, bleeding events, respiratory clinic follow-up and mortality.

Results 102 cases were identified of which 90 had acute PE. 81 sets of clinical notes were available. $24(29.6 \%)$ patients were managed as OP. This group was younger than those treated as IP $(59.30 \pm 3.84$ vs $64 \pm 2.12, p<0.05)$. The LOS was significantly shorter for OP: $(1.87 \pm 0.27$ vs $8.79 \pm 0.77$ days; $p<0.0001)$. There were no episodes of recurrent VTE or bleeding events at 90 days in either group, and only one readmission for anticoagulation related events (high INR; IP group). Three patients (3.7\%) died within 90 days (1 from sepsis, 1 from metastatic carcinoma and 1 from congestive cardiac failure) We identified a number of patients with low risk who were not treated as OP for a various reasons. All patients with OP management were subsequently followed by Respiratory team.

Conclusions Out patient management of diagnosed PE in a carefully selected group is practical, safe and decreases LOS using existing OP DVT services. The use of risk stratification assists identification of safe OP management.

\section{REFERENCES}

1. Aujesky D, et al. Lancet 2011;378:41-8.

2. Howard L, Salooja N. Lancet 2011;378:5-6.

\section{P7 ARE WE SCREENING SURVIVORS OF PULMONARY EMBOLISM (PE) FOR CHRONIC THROMBOEMBOLIC PULMONARY HYPERTENSION (CTEPH)?}

doi:10.1136/thoraxjnl-2011-201054c.7

M Wilczynska, K Taylor. Glan Clwyd Hospital, Rhyl, UK

Background Incidence of CTEPH following idiopathic PE has been reported as $4 \%$. The British Thoracic Society recommends that patients with massive or submassive PE should undergo echocardiography 6-12 weeks following the index event.

Aim To investigate local practice in the follow-up of patients with acute PE to devise management guidelines.

Methods A retrospective study of 110 patients diagnosed with acute $\mathrm{PE}$ at our hospital between 2007 and 2008 was conducted. Mean age was 68.6 years (range $27-100), 40(36 \%)$ were male and $18(16 \%)$ had previous venous thromboembolism. In 51 (46\%) patients $\mathrm{PE}$ was idiopathic.

Results All patients diagnosed with PE were normotensive and 27 (25\%) had in-patient echocardiography (ECHO). In 5 (18\%) patients scan confirmed RV dilatation and 2 of them had repeated ECHO within 2 months. Subsequently one patient was diagnosed with CTEPH and underwent pulmonary endarterectomy. In the group of patients with acute PE but without in-patient echocardiography 40 of 83 (48\%) received a follow-up appointment (mean 4 months) and $10(25 \%)$ had follow-up ECHO. Two more patients were diagnosed with CTEPH during this period (mean 34 months) with an overall incidence of $2.9 \%$.

Conclusion Recorded outcome, literature review and the BTS/ERS guidelines resulted in the development of local protocol for the screening acute PE survivors for CTEPH. [Abstract P7 figure 1]. 